\title{
ORIGINAL ARTICLE Dynamic landscape of the local translation at activated synapses
}

\author{
TM Khlebodarova ${ }^{1}$, VV Kogai ${ }^{2,3}$, EA Trifonova ${ }^{1}$ and VA Likhoshvai ${ }^{1,3}$
}

The mammalian target of rapamycin (mTOR) signaling pathway is the central regulator of cap-dependent translation at the synapse. Disturbances in mTOR pathway have been associated with several neurological diseases, such as autism and epilepsy. RNA-binding protein FMRP, a negative regulator of translation initiation, is one of the key components of the local translation system. Activation and inactivation of FMRP occurs via phosphorylation by S6 kinase and dephosphorylation by PP2A phosphatase, respectively. S6 kinase and PP2A phosphatase are activated in response to mGluR receptor stimulation through different signaling pathways and at different rates. The dynamic aspects of this system are poorly understood. We developed a mathematical model of FMRP-dependent regulation of postsynaptic density (PSD) protein synthesis in response to mGluR receptor stimulation and conducted in silico experiments to study the regulatory circuit functioning. The modeling results revealed the possibility of generating oscillatory (cyclic and quasi-cyclic), chaotic and even hyperchaotic dynamics of postsynaptic protein synthesis as well as the presence of multiple attractors in a wide range of parameters of the local translation system. The results suggest that autistic disorders associated with mTOR pathway hyperactivation may be due to impaired proteome stability associated with the formation of complex dynamic regimes of PSD protein synthesis in response to stimulation of mGluR receptors on the postsynaptic membrane of excitatory synapses on pyramidal hippocampal cells.

Molecular Psychiatry (2018) 23, 107-114; doi:10.1038/mp.2017.245; published online 5 December 2017

\section{INTRODUCTION}

Nowadays, there is convincing evidence that rapid and wellcoordinated changes in the quantitative and qualitative content of proteins at the synapse ensure its dynamic plasticity in response to external stimuli and underlie learning and memory. ${ }^{1-7}$ Disruption of local translation control at synapses has been associated with various neuropsychiatric diseases, including autism spectrum disorders, epilepsy, Parkinson's and Alzheimer's diseases (see reviews ${ }^{8-13}$ ), which are characterized by a shift in the balance of synaptic plasticity and lead to changes in behavior, cognitive abilities and memory.

The mammalian target of rapamycin (mTOR) pathway is the central regulator of cap-dependent translation at the synapse. Several recent studies have highlighted a pathogenic role of somatic mutations in genes of mTOR signaling pathway in the progression of certain forms of epilepsy and monogeneously inherited autism spectrum disorders, such as type 1 neurofibromatosis, Noonan syndrome, Costello syndrome, Kauden syndrome, Tuberous sclerosis, $\mathrm{X}$-chromosome fragility syndrome and Rett syndrome. ${ }^{13-19}$

It is believed that syndromic forms of autism account for up to $25 \%$ of the total autism spectrum ${ }^{20}$; however, microarray analysis of peripheral blood from patients with general/idiopathic autism has shown an abnormal activity of the signaling pathways associated with translation initiation ${ }^{21}$; and a direct measurement of Akt/mTOR pathway in cells isolated from the blood of children with non-syndromic autism demonstrated increased Akt/mTOR pathway activity as compared to the control group, suggesting a pathological role of mTOR pathway in the entire autism spectrum. 22

Fragile $X$ syndrome is the most common known syndromic form of autism, ${ }^{23,24}$ caused by expansion of the CGG repeat in the 5'untranslated region of FMR1 gene, leading to its transcriptional silencing. FMR1 gene encodes the RNA-binding protein FMRP (Fragile X Mental Retardation Protein), which is a regulator of mRNA stability and a negative regulator of local translation at synapses. $^{25}$

The peak FMR1 gene expression takes place during embryonic development, followed by mild reduction. The highest level of FMR1 gene expression has been recorded in the cells of hippocampus, cerebellum and cerebral cortex. ${ }^{26}$ In particular, FMRP controls the efficiency of dendritic mRNA translation in response to the stimulation of $\mathrm{mGluR}$ receptors (metabotropic glutamate receptors) on the postsynaptic membrane of excitatory synapses on pyramidal hippocampal cells. ${ }^{1,27}$ FMRP is the target of S6 kinase and PP2A phosphatase that are activated in response to stimulation of mGluR receptors. ${ }^{28,29}$ In a phosphorylated state, FMRP blocks translation by binding to MRNA, ribosomes and translation initiation factor elF4E. ${ }^{30-32}$ Dephosphorylation disrupts FMRP binding to its targets, which leads to the activation of mRNA translation.

FMRP target mRNAs encode proteins involved in mTOR signaling pathway (PI3K kinase, PTEN phosphatase, TSC2tuberous sclerosis complex-2, mTOR, PP2A phosphatase), receptor proteins (mGluR, NMDAR, AMPAR), proteins forming the postsynaptic membrane (NLGN, SHANK, PSD95), proteins of the

\footnotetext{
${ }^{1}$ Federal Research Center Institute of Cytology and Genetics, Siberian Branch of the Russian Academy of Sciences, Novosibirsk, Russia; ${ }^{2}$ Sobolev Institute of Mathematics, Siberian Branch of the Russian Academy of Sciences, Novosibirsk, Russia and ${ }^{3}$ Novosibirsk National Research State University, Novosibirsk, Russia. Correspondence: Dr TM Khlebodarova,

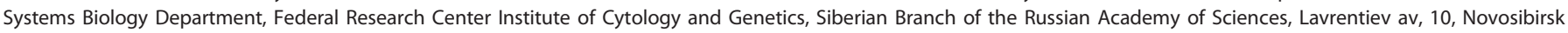
630090, Russian Federation.
}

E-mail: tamara@bionet.nsc.ru

Received 5 October 2017; accepted 9 October 2017; published online 5 December 2017 


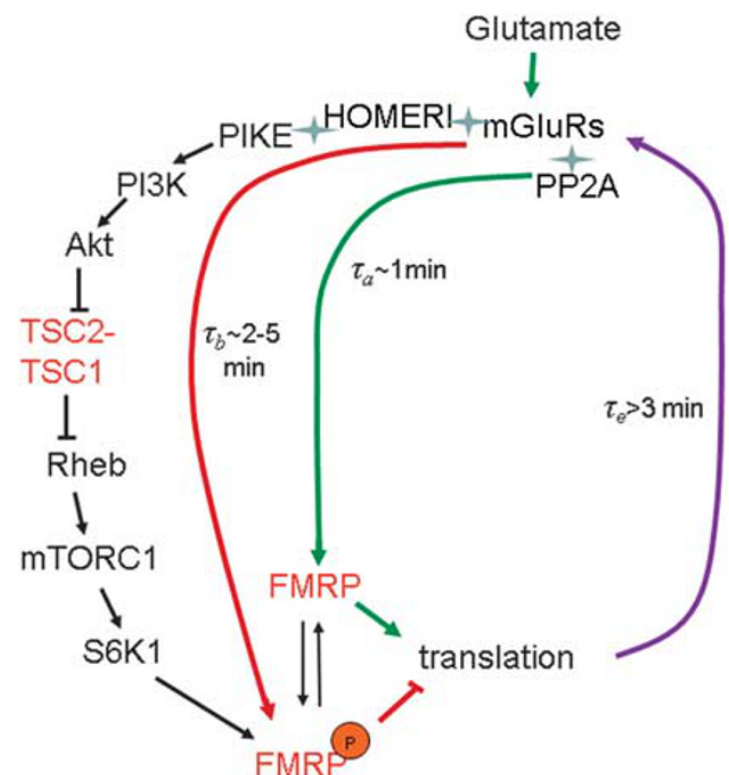

Figure 1. Signaling pathways regulating the activity of local capdependent translation at glutamatergic synapses in response to mGluR receptor stimulation. Names of proteins encoded by genes whose mutations are associated with neurodegenerative diseases, including autism, are marked with red color: $\tau_{e}$-total time of translation and inclusion of protein into the postsynaptic membrane, $\tau_{a}$ and $\tau_{b}$-delay parameters of the activation and suppression of translation, respectively.

ubiquitin-dependent protein degradation system (E3 ubiquitin ligase) and the FMR1 protein. ${ }^{24,30,33-36}$ These data demonstrate that FMRP plays a key role in the dynamic regulation of the synaptic proteome (see also reviews ${ }^{37,38}$ ).

The fact that stimulation of mGluR receptors is accompanied not only by rapid ( $1 \mathrm{~min}$ ) activation of PP2A phosphatase and, consequently, translation of FMRP-dependent mRNAs, but also by rapid activation of $\mathrm{S} 6$ kinase $(2-5 \mathrm{~min})$, which rephosphorylates FMRP and blocks the translation process, ${ }^{28,29}$ suggests dynamic interplay between the components of these signaling pathways at the activated synapse, ensuring its normal functioning.

Nowadays, the dynamic aspects of local translation at the activated synapse are poorly understood. It is possible that, in some cases, neuropsychiatric manifestations due to defects in synaptic function can be related to the peculiarities of the dynamic behavior of the local translation machinery. In the present work, we considered a simple regulatory contour of a local FMRP-dependent translation of postsynaptic density (PSD) proteins in response to stimulation of mGluR receptors on the postsynaptic membrane of an individual synapse and studied its dynamic properties by mathematical modeling. Structure of the investigated regulatory contour is illustrated in Figure 1.

In accordance with the analysis of dynamic properties of the model, there is a probability of observing complex dynamics of vibrational, chaotic and even hyperchaotic postsynaptic protein synthesis, depending on the ratio between the rate parameters for activation and suppression of local translation at the activated synapse. Moreover, the model has revealed parametric regions where coexistence of multiple attractors occur, which suggests the possibility of simultaneous existence of synapses with different dynamic properties. All these findings can hardly correlate with a stable proteome necessary for synaptic plasticity and memory formation. ${ }^{7,10}$ We do not exclude the possibility that these causes can underlie autistic and other types of disorders associated with activation of mTOR signaling pathway, which are manifested at higher hierarchical levels of the nervous system.

\section{Model}

The model describes the simplest genetic contour of FMRPdependent local translation of PSD proteins at glutamatergic synapses in response to mGluR receptor stimulation, depending on the ratio between the delay parameters of activation and suppression of translation (Figure 1). In order to study the internal properties of the regulatory contour, it is assumed in the model that a certain PSD protein density on the postsynaptic membrane of the activated synapse is maintained by de novo protein synthesis. Recycling of membrane proteins was not considered in the model. This simplification is justified for PSD proteins whose post-synaptic density in response to $\mathrm{mGluR}$ receptor stimulation is determined, among other things, by the balance between PSD protein synthesis and degradation. Such possibility has been demonstrated for PSD95, NMDAR and SHANK proteins with FMRP-

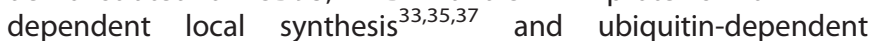
degradation ${ }^{39-41}$ at the activated synapse. It can also be true for mGluR protein itself, whose synthesis also depends on FRMP ${ }^{36}$ and internalization-on the process of its ubiquitination. ${ }^{42}$ In the model, the genetic contour (Figure 1) is described by the differential equation with delayed arguments

$$
\frac{\mathrm{d} a(t)}{\mathrm{d} t}=V_{i} \cdot F_{n}\left(a\left(t-\tau_{a e}\right), a\left(t-\tau_{b e}\right)\right)-k_{a} a(t)
$$

where $a(t)$, concentration of glutamate-specific receptors; $V_{i} \cdot F_{n}\left(a\left(t-\tau_{a e}\right), a\left(t-\tau_{b e}\right)\right)$, recovery rate for synaptic receptors; $k_{a} a(t)$, rate of membrane receptor utilization, $k_{a}$, rate constant for utilization; $F_{n}$, function describing regulation of local translation activation and suppression; $V_{i}$, parameter that sets the maximum initiation rate for local translation of receptors; $\tau_{a e}=\tau_{a}+\tau_{e}$ and $\tau_{b e}=\tau_{b}+\tau_{e}$, durations of the signal passage along the activation and translation contours, respectively; $\tau_{a}$, duration between signal generation on the receptor under the influence of glutamate molecule and signal realization in the form of FMRP dephosphorylation; $\tau_{b}$, duration between signal generation on the receptor under the influence of glutamate molecule and signal realization in the form of FMRP phosphorylation; $\tau_{e}$, total time of protein translation and its incorporation into the receptor complex.

The control function has the following form

$$
F_{n}(x, y)=\left[\frac{\left(\frac{x}{K_{a}}\right)^{h_{a}}}{1+\left(\frac{x}{K_{a}}\right)^{h_{a}}+\left(\frac{y}{K_{b}}\right)^{h_{b}}}\right]^{n}
$$

It belongs to the class of generalized Hill functions ${ }^{43}$ and, in the simplest phenomenological form, describes activation and suppression of local translation: as $x$ increases, the value of $F_{n}$ increases, and as $y$ increases, the value of $F_{n}$ decreases. Parameters $K_{a}$ and $K_{b}$ have the dimensionality of concentration and determine the impact of glutamate-specific signal on activation and suppression of local translation, respectively; parameters $h_{p}$ and $h_{b}$ are dimensionless Hill coefficients and determine the nonlinearity of the signal influence on the phosphorylation and dephosphorylation of FMRP, $n$ is the dimensionless Hill coefficient that determines the number of FMRP molecules dissociating with mRNA during activation process.

The evaluation of the model parameters $\left(\tau_{a}, \tau_{b}, \tau_{e}, h_{a}, h_{b}, n_{r}, V_{i}, K_{a r}\right.$ $K_{b}$ ) was carried out on the basis of a wide range of existing data on the structure and functions of glutamatergic synapses and mechanisms of local translation regulation in the activated synapse. ${ }^{28,29,31,32,36,44-54}$ Detailed description is given in Supplementary note 1. 

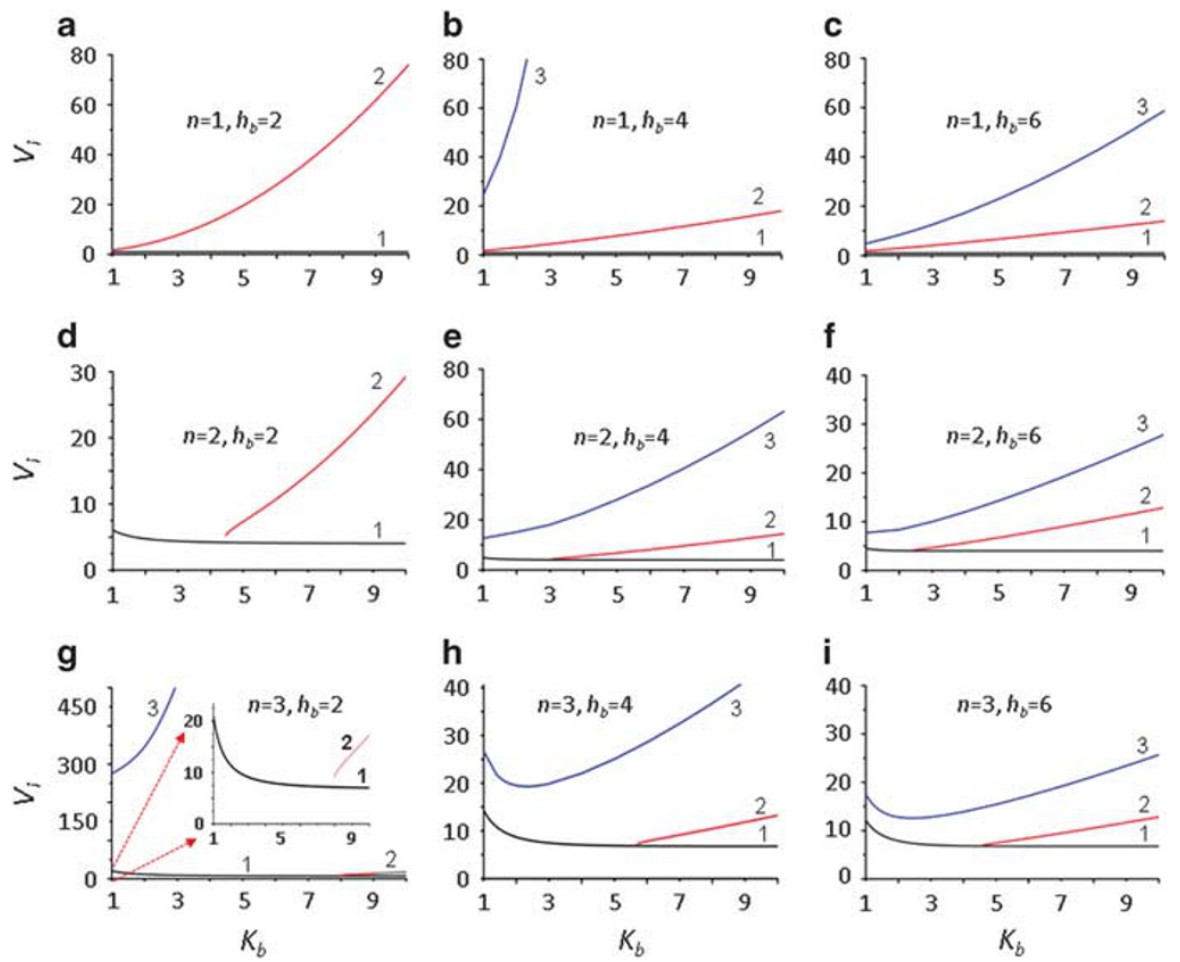

Figure 2. Areas of unconditional stability and predicted chaotic state of the maximal positive steady state solution of the model (1),(2) depending on the values of the efficiency constant for the suppression of local translation at the synapse $\left(K_{b}\right)$ and the maximal rate of receptor protein synthesis $(V)$.

\section{MATERIALS AND METHODS}

\section{Calculation method}

Integration of the equation was carried out by the steps method ${ }^{55}$ using an original program written in Fortran. Calculations were carried out on the computer complex of the Information and Computing Center of Novosibirsk State University (http://www.nusc.ru).

Stability analysis method for nonnegative steady-state solutions of equations (1) and (2)

Stability analysis method for a particular type of equations (1) and (2) has had no analogs in the literature and developed by us. Its detailed description and stability conditions for nonnegative steady-state solutions with nonzero values of the delayed arguments $\tau$ are given in Supplementary note 2 .

An empirical criterion for the existence of chaotic dynamics in the delay differential equation of a special type

To estimate the values of the parameters $V_{i r}, k_{a}, K_{a}, K_{b}, h_{p}$ and $h_{b}$, for which chaotic dynamics is predicted for equation (1), an empirical criterion was applied. $^{56}$

Let us give its formulation. Let there be given an equation with delayed argument of the following form

$$
\frac{\mathrm{d} p(t)}{\mathrm{d} t}=f(p(t-\tau))-p(t) g(p(t-\tau))
$$

Let us consider a chaotic one-dimensional mapping $x_{m+1}=G\left(x_{m}\right), \quad G(x)=f(x) / g(x)$ of a segment into itself. Then, equation (5) would have a strange attractor for certain value of $\tau$. For equations (1) and (2), we have $G(x)=\left(V i / k_{a}\right) F_{n}(x, x)$.

To estimate the degree of chaos for the one-dimensional mapping $x_{m+1}=G\left(x_{m}\right)$, we applied the Li-Yorke theorem, according to which the existence of a cycle of length 3 means that this mapping is chaotic. ${ }^{57}$ There is no corresponding criterion for unequal values of the delayed arguments $\tau_{a e} \leqslant \tau_{b e}$. Therefore, the chaotic dynamics was studied by numerical analysis, which was determined using three criteria: the sensitivity of solutions with respect to initial data, the positivity of the value of the largest Lyapunov exponent and the special form of the Poincare map generated by the solution. Let us give a brief description.

Chaos criterion for the oscillatory dynamics

The sensitivity of the oscillatory dynamics was calculated as the difference between two trajectories of one variable, calculated by two identical models that start at zero time point with the initial functions that vary by a 'small' value. If, as we iterate over values, the difference became comparable with the fluctuation amplitude, the dynamics was concluded to be sensitive to initial data.

\section{Lyapunov exponents}

To quantify the instability of the solution with respect to initial data, Lyapunov exponents were used that characterize the degree of exponential growth (or decay) of disturbances near the attractor trajectory. A spectrum of Lyapunov exponents has a dimensionality of the phase space. For systems of equations with delayed arguments, for which the phase space is infinite, the number of Lyapunov exponents is also infinite. However, only a few senior Lyapunov exponents have a significant impact. Knowing the spectrum of Lyapunov exponents enables to solely identify the motion nature in the system. Presence of at least one positive Lyapunov exponent is a measure of chaos. If a chaotic dynamics had two or more positive Lyapunov exponents, such dynamics was identified to be hyperchaotic. ${ }^{58}$

Method for calculating the largest Lyapunov exponent for the delay equation is a modification of the Benettin method ${ }^{59,60}$ and was previously described ${ }^{61,62}$

\section{Poincaré map}

A characteristic form of the Poincaré map (the so-called succession map) allows to explicitly identify the motion pattern in the system. Thus, a cyclic trajectory generates a Poincaré map consisting of a finite number of points through which the trajectory passes with certain regularity. A quasicyclic trajectory is mapped onto a plane as a set of closed curves. The Poincaré map corresponding to a strange attractor represents an unordered infinite set of points. The method for constructing the Poincaré map has been described earlier. ${ }^{61,62}$ 

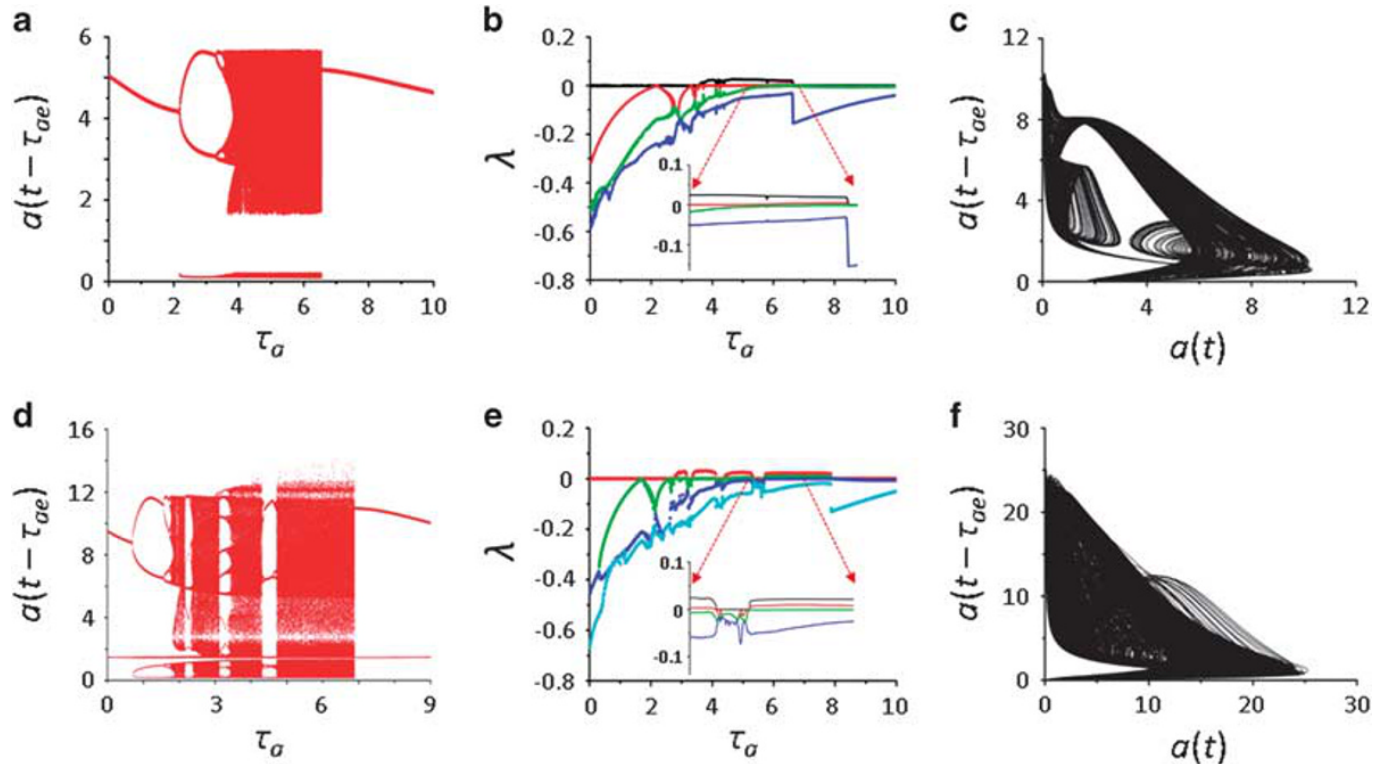

Figure 3. Solutions of equations $(1,2)$ for a similar proportion of delay parameters of activation and suppression of translation $\tau_{a}=\tau_{b}=\tau_{\text {. }}(\mathbf{a}, \mathbf{d})$ bifurcation diagrams constructed from the intersection of the trajectory $\left(a(t), a\left(t-\tau_{a e}\right)\right)$ and the Poincare plane $a(t)=5$; (b,e) values of the largest Lyapunov exponents depending on the delay parameter $\tau_{a}$; (c) chaos at $\tau_{a}=\tau_{b}=4$; (f) hyperchaos at $\tau_{a}=\tau_{b}=7$, the largest Lyapunov exponents: $\lambda_{1}=0.02127, \lambda_{2}=0.0078$. Parameter values: $n=1, h_{b}=4, V_{i}=30$ (a-c), $n=3, h_{b}=2, V_{i}=700$ (d-f), values of the remaining parameters: $k_{a}=1, K_{a}=1, K_{b}=1, h_{a}=1, \tau_{e}=3$.

\section{RESULTS}

In this section, we analyzed a model, in which the time was measured in minutes and the concentration-in units equal to $K_{a}$. The transition to this concentration unit was carried out by the standard procedure for changing the variables $a(t):=a(t) / K_{a}, V_{i}:=$ $V_{i} / K_{a}, K_{b}:=K_{b} / K_{a}$. For the sake of convenience, we left the same notation of the variable and the parameters. Also, according to the experimental data (see the section 'Estimation of the model parameters'), we fixed $k_{a}=1 \mathrm{~min}^{-1}$ and $h_{a}=1$.

Analysis of the steady-state stability and chaotic potential stability in model (1),(2)

The results of the positive steady-state stability analysis depending on the values of the parameters $V_{i}$ and $K_{b}$ are presented in Figure 2. Calculations were made for nine variants of $h_{b}$ and $n$ values, which were changed in discrete ranges: $h_{b}=2,4,6$ and $n=1,2,3$.

For each variant, the area of existence of positive steady states of model (1),(2) lies above curve 1. There are no positive steady states of the model in the area below curve 1 . When $n=1$, curve 1 represents the line $V_{i}=1$ (Figures $2 \mathrm{a}-\mathrm{c}$ ), that is, it is exactly equal to the physiological minimum of this parameter, defined in the section 'Estimation of the model parameters', Supplementary note 1. When $n=2,3$, the boundary of existence of a positive steady state (curve 1 ) is a function monotonically decreasing from $K_{b}$. When $n=2$, the function tends to the limit $V_{i}=4$ from above, and when $n=3$ it tends to the limit $V_{i}=6.75$. That is, with an increase of $n$, the minimum value of $V_{i}$ that determines the applicability of the model to the target object increases.

Areas between curves 1 and 2 represent regions of unconditional stability, since maximum steady states in this areas remain stable for any values of delayed arguments, $\tau_{b e} \geqslant \tau_{a e} \geqslant 0$. When $n=1$ (Figures $2 a-c$ ), the region of unconditional stability exists over the entire range of the parameter $K_{b}$ from 1 to 10 . When $n=2$ (Figures $2 \mathrm{~d}-\mathrm{f}$ ) and $n=3$ (Figures $2 \mathrm{~g}-\mathrm{i}$ ), the boundary of the unconditional stability region shifts to the right from the origin and this region is absent for the values of the parameter $K_{b}$ lying to the left of this boundary. Qualitative behavior of the unconditional stability region is invariable for all values of $n$. It monotonically increases with increasing value of $K_{b}$ and for $n=2,3$ its boundary shifts to the origin when the value of parameter $h_{b}$ increases. That is, unconditional stability region increases with the decrease in the efficiency of functioning of the FMRP-dependent activation/suppression of translation.

Areas above curve 2 represent regions of conditional stability, since within this region maximal steady states lose stability for certain values of delayed arguments, $\tau_{b e} \geqslant \tau_{a e} \geqslant 0$.

Curve 3 represents the boundary separating the region of conditional stability into two subregions. There is a parametric zone above curve 3 in which, according to the empirical criterion, ${ }^{56}$ a chaotic dynamics is predicted. Below these curves, a chaotic dynamics is not predicted. It should be noted that the share of 'chaotic' zone in the conditional stability region essentially depends on the parameters of the model and increases markedly with the increase of $n \times h_{b}$. The results of the analysis of the parametric zone in which chaotic dynamics is predicted for model (1),(2) are presented below.

Analysis of the dynamics complexity for particular parameter values

Regulatory delays play an important role in the formation of chaotic dynamics. ${ }^{61-64}$ But the empirical criterion does not provide information on the values of delayed arguments. Its implementation only guarantees the presence of certain equal values $\tau_{a e}=\tau_{b e}$, for which the chaotic dynamics is observed. Moreover, there is yet no criterion for predicting chaotic dynamics for different values $\tau_{a e}<\tau_{b e}$. In this case, the only analytical method is a numerical experiment. However, a large combinatorial networks of possible combinations of parameter values do not allow for the detailed numerical analysis in the entire physiological range of their variation. In this section, we have considered two polar variants of the values of Hill coefficients $n$ and $h_{b}: n=1, h_{b}=4$ and $n=3$, $h_{b}=2$, which reflect the contrast variants of the suppression of local translation at the synapse in the range of physiological 

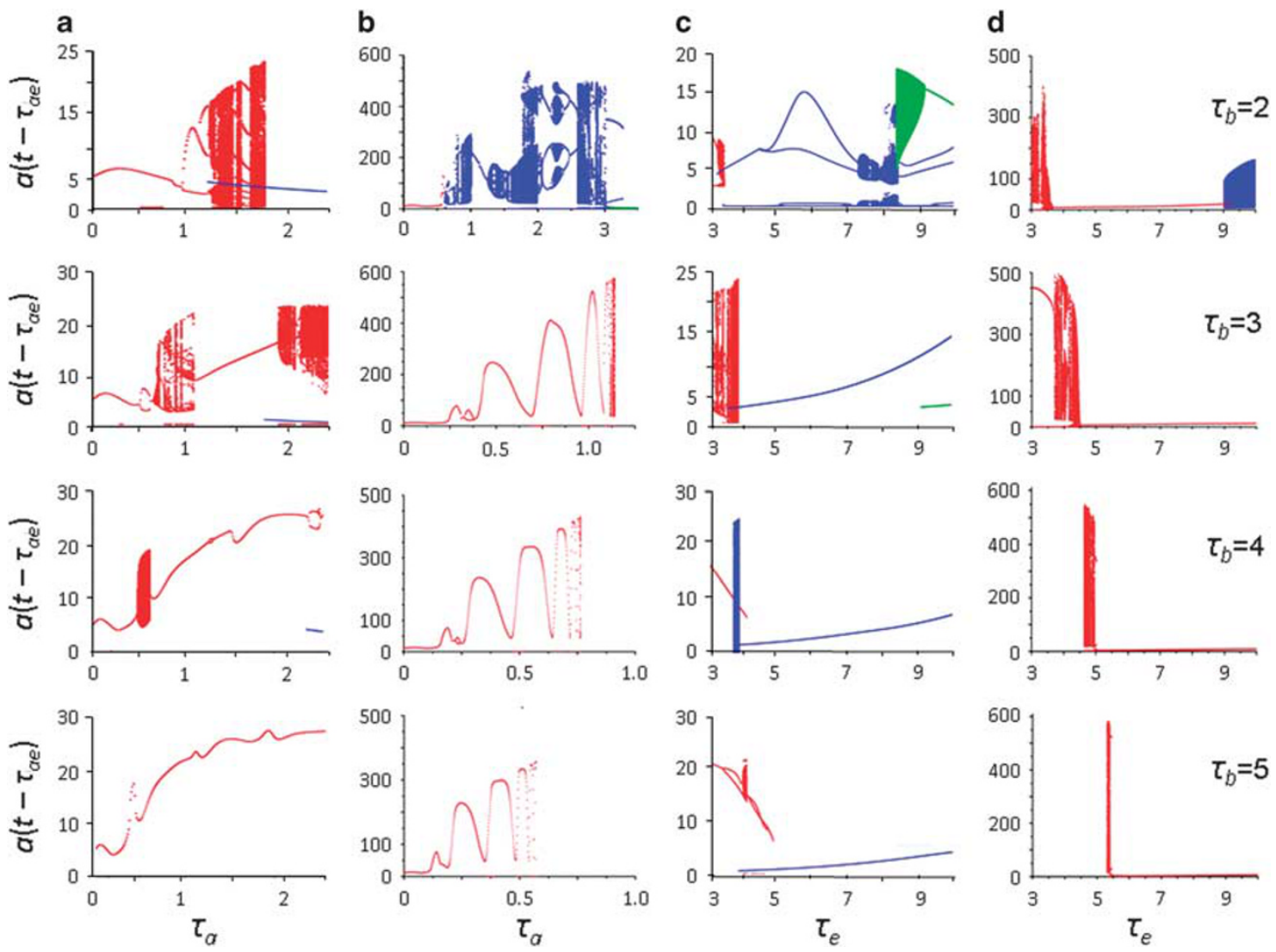

Figure 4. Solutions of equations (1) and (2) depending on the delay parameters: bifurcation diagrams constructed from the intersection of the trajectory $\left(a(t), a\left(t-\tau_{a e}\right)\right)$ and the Poincaré planes $a(t)=5(\mathbf{a}, \mathbf{c})$ and $a(t)=10(\mathbf{b}, \mathbf{d})$. Columns $(\mathbf{a}),(\mathbf{c})$ represent a variant $n=1, h_{b}=4, V_{i}=30, \tau_{e}=3(\mathbf{a}), \tau_{a}=1$ (c); columns (b),(d) variant $n=3, h_{b}=2, V_{i}=700, \tau_{e}=3(\mathbf{c}), \tau_{a}=1$ (d). Value of the parameter $\tau_{b}$ for each row is shown on the right-hand side of the figure. Values of the remaining parameters $k_{a}=1, K_{a}=1, K_{b}=1, h_{a}=1$. Different branches of solutions (attractors) are shown in different colors.

values defined in the section 'Estimation of the model parameters', Supplementary note 1 . Calculations for the variant $n=1$, $h_{b}=4$ were carried out for $V_{i}=30$, and for the variant $n=3, h_{b}=2$ -for $V_{i}=700$. Both variants, according to the empirical criterion, ${ }^{56}$ are located in areas of predicted chaotic dynamics.

Numerical calculations shown in Figure 3 confirmed that model (1),(2) exhibits chaotic dynamics in both variants, at least for $\sim 5<\tau_{a e}=\tau_{b e}<\sim 9.5$. This is evidenced by the bifurcation diagrams shown in Figures $3 a$ and $d$. It is recognized that in both cases the transition to chaos is carried out through a cascade of oscillation period doubling, that is, according to the Feigenbaum scenario. ${ }^{65,66}$ This scenario is one of the most frequently observed scenarios of transition to chaos and is described, in particular, in models of dynamic electrical activity of neurons and pancreatic $\beta$ cells, ${ }^{67,68}$ in models of intracellular oscillations of $\mathrm{Ca}^{2+}$ and $\mathrm{NADH}$ concentrations, ${ }^{69,70}$ and also in models of alternative splicing. ${ }^{64,71}$ It is also seen in Figure $3 d$ that chaotic regions intersect with zones of regular cyclic dynamics. The existence of hyperchaotic solutions for $n=3, h_{b}=2$ (Figure $3 f$ ) is evidenced by the presence of two positive Lyapunov exponents that emerge in the range of values $\tau_{a}=6 \div 8$ (Figure $3 \mathrm{e}$ ).

Dynamic properties of model (1),(2) depending on the ratio of the delay parameters $\tau_{b} / \tau_{a}>1$

Since it is known that for the normal activated synapse values of the parameters $\tau_{a}$ and $\tau_{b}$ are presumably not equal, but differ by a factor of $2-5,{ }^{28,29}$ and application of the empirical criterion does not provide information on the chaotic potential of the model for unequal values of delayed arguments, in this section, we carried out a direct numerical study of the dynamic properties of the above shown variants of model (1),(2) for $\tau_{b e}>\tau_{a e}$.

The results of the analysis are shown in Figure 4.

It can be seen that in the entire range of delay values, there are no stable steady states and, on the contrary, a complex pattern of oscillatory dynamics is observed, namely periodic, quasiperiodic or chaotic; and there are existence regions for multiple attractors. It should also be noted that an increase in the $\tau_{b e} / \tau_{a e}$ ratio, although it did not exclude the existence of zones of chaotic attractor, generally led to an increase in the areas with cyclic dynamics (Figure 4, compare rows from top to bottom).

A concrete example demonstrating the existence in the system of local translation of multiple attractors in one parametric area is given in Supplementary Figure S2.

Dynamic properties of model (1),(2) depending on the values of parameters $h_{b}$ and $\tau_{e}$

Parameter $h_{b}$ determines the degree of nonlinearity of the mechanism behind realization of mTOR signaling; and parameter $\tau_{e}$ represents the total time of protein synthesis and inclusion of the newly synthesized protein into the PSD.

From the results presented in Figure 4, it can be seen that the particular values of given parameters significantly affect the dynamic behavior of the system. Therefore, we carried out an extended analysis of the joint impact of the parameters $h_{b}$ and $\tau_{e}$ on the operating modes of model (1),(2). In the analysis, we only considered the case where $n=1$, which corresponds to the currently dominant hypothesis on the mechanisms of translation 


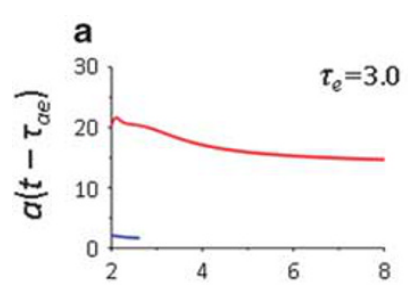

\section{b}

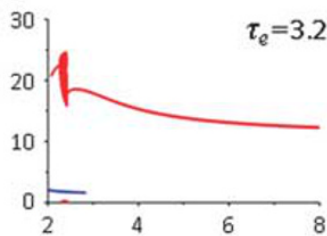

f
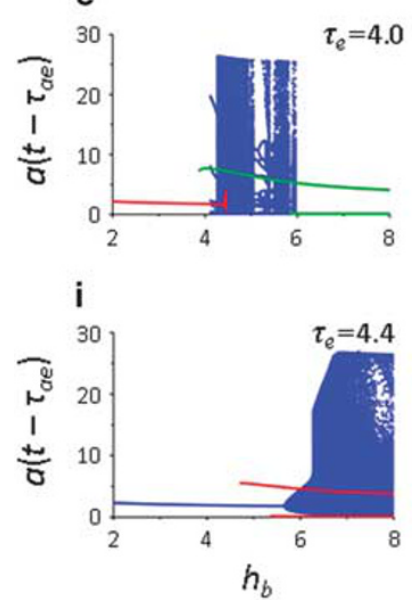

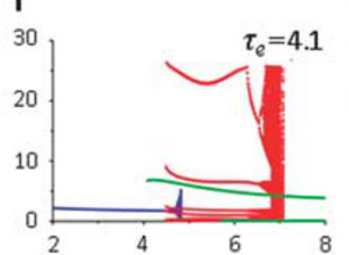

j

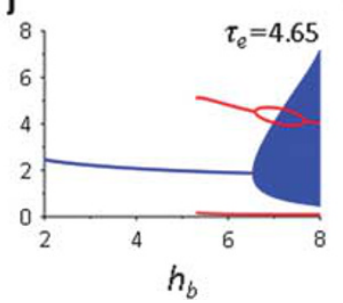

c

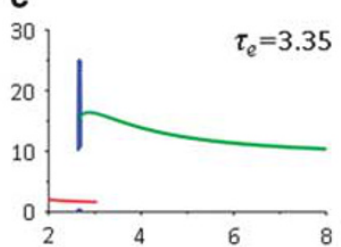

\section{g}

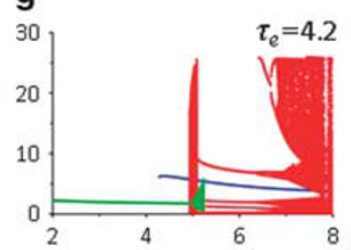

k

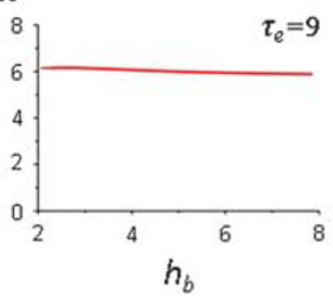

d

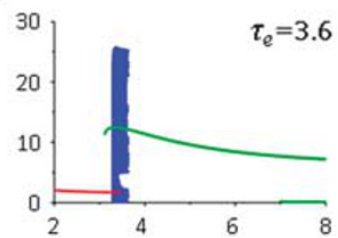

h

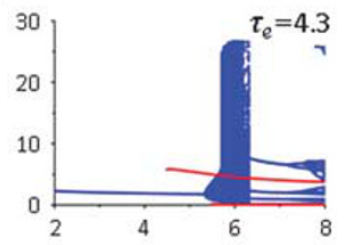

I

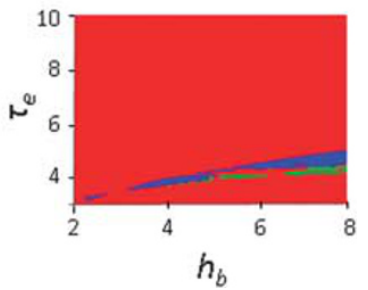

Figure 5. Modes of functioning of the model (1),(2) implemented for the ratio of delay parameters $\tau_{b} / \tau_{a}=4$ and depending on the complexity of the mTOR signaling pathway $\left(h_{b}\right)$. (a)-(k) bifurcation diagrams constructed from the intersection of the trajectory $\left(a(t)\right.$, $\left.a\left(t-\tau_{a e}\right)\right)$ and the Poincaré plane $a(t)=5$. Different attractors are shown in different colors. (I) solutions of the model (1), (2) in the value space $\tau_{e}=3-10$ and $h_{b}=2-8$. Red color shows periodic solutions, green-quasiperiodic solutions, and blue-chaotic solutions. Values of the remaining parameters: $k_{a}=1, K_{a}=1, K_{b}=1, h_{a}=1, V_{i}=30, n=1, \tau_{a}=1$.

regulation through FMRP, ${ }^{27,67}$ as well as fixed ratio $\tau_{b} / \tau_{a}=4$, which is also physiologically justified. ${ }^{24,25}$

The results of the analysis are summarized in Figure 5.

From Figure $5 \mathrm{l}$ it follows that for $\tau_{e}>5$, only cyclic type of dynamic behavior of model (1),(2) is observed in the whole range of variation $2 \leqslant h_{b} \leqslant 8$. At the same time, for $\tau_{e}<5$, the nature of the dynamics depends considerably on the value of $h_{b}$. Therefore, all types of dynamics are detected in the region $3.0 \leqslant \tau_{e} \leqslant 5$ : cyclic, quasicyclic and chaotic (Figure 5l), and the transition from one type of behavior of model (1),(2) to another was extremely sensitive to the value of the parameter $\tau_{e}$ (Figures $5 \mathrm{a}-\mathrm{j}$ ).

Additionally, we note that multiple attractors were observed for certain values of the parameters $\tau_{e}$ and $h_{b}$, which are illustrated in different colors in Figures $5 a-j$. The phenomenon of the multiplicity of attractors, which we also observed in Figure 4, demonstrates the feasibility of diverse dynamic states of the local translation system at various activated synapses. However, this phenomenon was observed only for relatively low values of the parameter $3<\tau_{e}<5$ and with increasing $\tau_{e}$ the area of the attractor multiplicity shifted to the area with high values of $h_{b}$, so that for $\tau_{e}=9$ only single cyclic attractors were found in the studied range of $h_{b}$ (Figure $5 \mathrm{k}$ ).

Thus, the studies presented above showed that the revealed complex dynamics (stationary, cyclic, quasicyclic, chaotic, hyperchaotic) of model (1),(2) was carried out during the implementation of the positive and negative regulatory loops controlling local translation at real synapses. ${ }^{28,29}$

\section{DISCUSSION}

Existing views on the importance of a stable proteome for the formation of synaptic plasticity and associated processes of learning and memory (see reviews $s^{4,7,10}$ ) reveal the significant role for dysregulation of postsynaptic protein synthesis, recyclization and degradation at the activated synapse in the pathogenesis of neurological diseases (autism, epilepsy, and so on). In this study, given certain experimental data, ${ }^{28,29}$ we focused only on the dynamic aspects of functioning of the translation system. It allowed us to single out the simplest regulatory contour and investigate the subtle dynamic relationships between activation and suppression of local translation at glutamatergic synapses in response to their activation. As shown above, these relationships can cause steady-state instabilities of the local translation system and formation of a complex dynamics of postsynaptic protein synthesis in a considerable part of the physiological range. Both cyclic and chaotic dynamics of FMRP-dependent postsynaptic protein synthesis result from a decrease in the activation ratio between S6 kinase and PP2A phosphatase, the two regulators of FMRP phosphorylation, playing a key role in controlling local translation at the synapse. This is possible both as a result of increased activity of mTOR signaling pathway controlling the activity of S6 kinase, and as a result of decreased PP2A phosphatase activity. Therefore, it is not surprising that one of the indications of epilepsy and autistic states is increased mTOR activity, ${ }^{12,15,16,18,19}$ which can occur both as a result of mutations and various external influences.

Owing to the simplicity of the presented model, we cannot claim that the model completely reflects all existing interrelations, but one thing is certain: mechanisms that regulate local translation contain destabilizing elements. The very existence of negative and positive regulatory loops in the local translation system is one of the factors sufficient for the formation of complex, chaotic dynamics. ${ }^{61-64,71}$

Another problem is, in what parametric region can this dynamics be implemented and what additional conditions are evolutionarily created by the cell to stabilize the function of the synapse? There are no answers to these questions yet. 
First of all, due to the lack of sufficiently detailed data on the mechanisms of synaptic function, it is impossible to easily determine the parametric boundaries of chaos generation. In our model, along with insufficient information on the values of $K_{a}$ $K_{b}$ and $V_{i}$ constants, the lack of reliable data on the number of glutamatergic receptors at the PSD activated synapse is the main reason for parametric uncertainty. Existing estimates give a spread of 4 to 1200 molecules per PSD. ${ }^{5-54}$ The uncertainty of the data is such that almost no part of the parametric area can be classified as not corresponding to reality.

To illustrate, let us consider the calculation shown in Figure 3c. In this calculation, the dynamics is chaotic and $a(t)$ ranges from 0.25 to 210 dimensionless units (ratio between the current number of receptors on the membrane and the value of the $K_{a}$ parameter). If we set the value of $K_{a}$, which is unknown, considering that the value of $a(t)$ lies in the experimental boundaries (for example, $K_{a}=5$ molecules per membrane), then the value of $a(t)$ varies from 1.25 to 1050 molecules per membrane. It is evident that the obtained variation corresponds well with the region between the minimum and maximum estimates: 4-1200 molecules/membrane. ${ }^{52-54}$

As for the second problem, it requires not only parametric certainty, but also additional studies on the structural complexity of the system and its impact on the synaptic stability. In our model, we analyzed only two such parameters, $\left(h_{b}\right)$ and $(n)$, related to the regulation of FMRP-dependent translation via mTOR signaling pathway. The obtained results clearly indicate that mechanisms of maintaining proteome stability at the activated synapse, that are critical for the formation of synaptic plasticity, 7,10 are directly related to the mechanisms of suppression of FMRPdependent translation at the activated synapse.

In conclusion, it should be noted that we have obtained theoretical data on the dynamic regimes of functioning of the local translation at the activated synapse, which, however, due to uncertainty in a number of parameters, do not allow us to clearly specify which regimes are implemented in the synapses in norm and pathology. This is a very important issue related to understanding mechanisms of the onset of neuropsychiatric diseases due to disturbed local translation at the synapse. ${ }^{1,4,15-19,22}$ Unfortunately, currently existing quantitative data on the structure and function of synapses do not allow to answer this question. This makes the detailed analysis of the biochemical characteristics and parameters of functioning of the synapses extremely important.

\section{CONFLICT OF INTEREST}

The authors declare no conflict of interest.

\section{ACKNOWLEDGMENTS}

This work was partly supported by the Russian State Budget (project No 0324-20160008) and by the RFBR (project No 16-01-00237a). We thank Tatyana Kalymbetova for the translation of an article from Russian to English, and Alena Moiseenko for the information support for this study.

\section{REFERENCES}

1 Huber KM, Kayser MS, Bear MF. Role for rapid dendritic protein synthesis in hippocampal mGluR-dependent long-term depression. Science 2000; 288: 1254-1257.

2 Hou L, Antion MD, Hu D, Spencer CM, Paylor R, Klann E. Dynamic translational and proteasomal regulation of fragile $\mathrm{X}$ mental retardation protein controls mGluRdependent long-term depression. Neuron 2006; 51: 441-454.

3 Costa-Mattioli M, Sossin WS, Klann E, Sonenberg N. Translational control of longlasting synaptic plasticity and memory. Neuron 2009; 61: 10-26.

4 Cajigas IJ, Will T, Schuman EM. Protein homeostasis and synaptic plasticity. EMBO J 2010; 29: 2746-2752.
5 Santini E, Huynh TN, Klann E. Mechanisms of translation control underlying longlasting synaptic plasticity and the consolidation of long-term memory. Prog $\mathrm{Mol}$ Biol Transl Sci 2014; 122: 131-167.

6 Rosenberg T, Gal-Ben-Ari S, Dieterich DC, Kreutz MR, Ziv NE, Gundelfinger ED et al. The roles of protein expression in synaptic plasticity and memory consolidation. Front Mol Neurosci 2014; 7: 86.

7 Louros SR, Osterweil EK. Perturbed proteostasis in autism spectrum disorders. J Neurochem 2016; 139: 1081-1092.

8 Buffington SA, Huang W, Costa-Mattioli M. Translational control in synaptic plasticity and cognitive dysfunction. Annu Rev Neurosci 2014; 37: 17-38.

9 Gkogkas CG, Sonenberg N. Translational control and autism-like behaviors. Cell Logist 2013; 3: e24551.

10 Klein ME, Monday H, Jordan BA. Proteostasis and rna binding proteins in synaptic plasticity and in the pathogenesis of neuropsychiatric disorders. Neural Plast 2016; 2016: 3857934.

11 Martin I. Decoding Parkinson's disease pathogenesis: the role of deregulated mRNA translation. J Parkinsons Dis 2016; 6: 17-27.

12 Cai Z, Chen G, He W, Xiao M, Yan L. Activation of mTOR: a culprit of Alzheimer's disease? Neuropsychiatr Dis Treat 2015; 11: 1015-1030.

13 Trifonova EA, Khlebodarova TM, Gruntenko NE. Molecular mechanisms of autism as a form of synaptic dysfunction. Vavilov J Genet Breed 2016; 20: 959-967, (Russian).

$14 \mathrm{Cho} \mathrm{CH}$. Frontier of epilepsy research-mTOR signaling pathway. Exp Mol Med 2011; 43: 231-274.

15 Meng XF, Yu JT, Song JH, Chi S, Tan L. Role of the mTOR signaling pathway in epilepsy. J Neurol Sci 2013; 332: 4-15.

16 Won H, Mah W, Kim E. Autism spectrum disorder causes, mechanisms, and treatments: focus on neuronal synapses. Front Mol Neurosci 2013; 6: 19.

17 Goldani AA, Downs SR, Widjaja F, Lawton B, Hendren RL. Biomarkers in autism. Front Psychiatry 2014; 5: 100.

18 Lipton JO, Sahin M. The neurology of mTOR. Neuron 2014; 84: 275-291.

19 Huber KM, Klann E, Costa-Mattioli M, Zukin RS. Dysregulation of mammalian target of rapamycin signaling in mouse models of autism. J Neurosci 2015; 35: 13836-13842.

$20 \mathrm{Yoo} \mathrm{H}$. Genetics of autism spectrum disorder: current status and possible clinical applications. Exp Neurobiol 2015; 24: 257-272.

21 Pramparo T, Pierce K, Lombardo MV, Carter Barnes C, Marinero S, Ahrens-Barbeau $C$ et al. Prediction of autism by translation and immune/inflammation coexpressed genes in toddlers from pediatric community practice. JAMA Psychiatry 2015; 72: 386-394.

22 Onore C, Yang H, Van de Water J, Ashwood P. Dynamic Akt/mTOR signaling in children with autism spectrum disorder. Front Pediatr 2017; 5: 43.

23 Bassell GJ, Warren ST. Fragile $X$ syndrome: loss of local mRNA regulation alters synaptic development and function. Neuron 2008; 60: 201-214.

24 Sharma A, Hoeffer CA, Takayasu Y, Miyawaki T, McBride SM, Klann E et al. Dysregulation of mTOR signaling in fragile $X$ syndrome. J Neurosci 2010; 30: 694-702.

25 Feng Y, Absher D, Eberhart DE, Brown V, Malter HE, Warren ST. FMRP associates with polyribosomes as an mRNP, and the I304N mutation of severe fragile $X$ syndrome abolishes this association. Mol Cell 1997; 1: 109-118.

26 Kleijer KTE, Huguet G, Tastet J, Bourgeron T, Burbach JPH. Anatomy and cell biology of autism spectrum disorder: lessons from human genetics. Adv Anat Embryol Cell Biol 2017; 224: 1-25.

27 Chen $E$, Joseph S. Fragile $X$ mental retardation protein: a paradigm for translational control by RNA-binding proteins. Biochimie 2015; 114: 147-154.

28 Narayanan U, Nalavadi V, Nakamoto M, Pallas DC, Ceman S, Bassell GJ et al. FMRP phosphorylation reveals an immediate-early signaling pathway triggered by group I mGluR and mediated by PP2A. J Neurosci 2007; 27: 14349-14357.

29 Narayanan U, Nalavadi V, Nakamoto M, Thomas G, Ceman S, Bassell GJ et al. S6K1 phosphorylates and regulates fragile $X$ mental retardation protein (FMRP) with the neuronal protein synthesis-dependent mammalian target of rapamycin (mTOR) signaling cascade. J Biol Chem 2008; 283: 18478-18482.

30 Brown V, Small K, Lakkis L, Feng Y, Gunter C, Wilkinson KD et al. Purified recombinant Fmrp exhibits selective RNA binding as an intrinsic property of the fragile X mental retardation protein. J Biol Chem 1998; 273: 15521-15527.

31 Napoli I, Mercaldo V, Boyl PP, Eleuteri B, Zalfa F, De Rubeis S et al. The fragile X syndrome protein represses activity-dependent translation through CYFIP1, a new 4E-BP. Cell 2008; 134: 1042-1054.

32 Chen E, Sharma MR, Shi X, Agrawal RK, Joseph S. Fragile X mental retardation protein regulates translation by binding directly to the ribosome. Mol Cell 2014; 54: 407-417.

33 Muddashetty RS, Kelić S, Gross C, Xu M, Bassell GJ. Dysregulated metabotropic glutamate receptor-dependent translation of AMPA receptor and postsynaptic density-95 mRNAs at synapses in a mouse model of fragile $X$ syndrome. J Neurosci 2007; 27: 5338-5348. 
34 Gross C, Nakamoto M, Yao X, Chan CB, Yim SY, Ye K et al. Excess phosphoinositide 3-kinase subunit synthesis and activity as a novel therapeutic target in fragile $X$ syndrome. J Neurosci 2010; 30: 10624-10638.

35 Ascano M Jr, Mukherjee N, Bandaru P, Miller JB, Nusbaum JD, Corcoran DL et al. FMRP targets distinct mRNA sequence elements to regulate protein expression. Nature 2012; 492: 382-386.

36 Darnell JC, Van Driesche SJ, Zhang C, Hung KY, Mele A, Fraser CE et al. FMRP stalls ribosomal translocation on mRNAs linked to synaptic function and autism. Cell 2011; 146: 247-261.

37 Zukin RS, Richter JD, Bagni C. Signals, synapses, and synthesis: how new proteins control plasticity. Front Neural Circuits 2009; 3: 14.

38 lacoangeli $\mathrm{A}$, Tiedge $\mathrm{H}$. Translational control at the synapse: role of RNA regulators. Trends Biochem Sci 2013; 38: 47-55.

39 Colledge M, Snyder EM, Crozier RA, Soderling JA, Jin Y, Langeberg LK et al. Ubiquitination regulates PSD-95 degradation and AMPA receptor surface expression. Neuron 2003; 40: 595-607.

40 Ehlers MD. Activity level controls postsynaptic composition and signaling via the ubiquitin-proteasome system. Nature Neuroscience 2003; 6: 231-242.

41 Kato A, Rouach N, Nicoll RA, Bredt DS. Activity-dependent NMDA receptor degradation mediated by retrotranslocation and ubiquitination. Proc Natl Acad Sci USA 2005; 102: 5600-5605.

42 Gulia R, Sharma R, Bhattacharyya S. A critical role for ubiquitination in the endocytosis of glutamate receptors. J Biol Chem 2017; 292: 1426-1437.

43 Likhoshvai V, Ratushny A. Generalized hill function method for modeling molecular processes. J Bioinform Comput Biol 2007; 5: 521-531.

44 Boström K, Wettesten M, Borén J, Bondjers G, Wiklund O, Olofsson SO. Pulsechase studies of the synthesis and intracellular transport of apolipoprotein B-100 in Hep G2 cells. J Biol Chem 1986; 261: 13800-13806.

45 Ingolia NT, Lareau LF, Weissman JS. Ribosome profiling of mouse embryonic stem cells reveals the complexity and dynamics of mammalian proteomes. Cell 2011; 147: 789-802.

46 Sharma K, Fong DK, Craig AM. Postsynaptic protein mobility in dendritic spines: long-term regulation by synaptic NMDA receptor activation. Mol Cell Neurosci 2006; 31: 702-712.

47 Bartley CM, O'Keefe RA, Blice-Baum A, Mihailescu MR, Gong X, Miyares L et al. Mammalian FMRP S499 is phosphorylated by CK2 and promotes secondary phosphorylation of FMRP. eNeuro 2016; 3: 6 .

48 Ceman S, O'Donnell WT, Reed M, Patton S, Pohl J, Warren ST. Phosphorylation influences the translation state of FMRP-associated polyribosomes. Hum Mol Genet 2003; 12: 3295-3305.

49 Bhaskar PT, Hay N. The two TORCs and Akt. Dev Cell 2007; 12: 487-502.

50 Majumder P, Chu JF, Chatterjee B, Swamy KB, Shen CJ. Co-regulation of mRNA translation by TDP-43 and Fragile X Syndrome protein FMRP. Acta Neuropathol 2016; 132: 721-738.

51 Pandey S, Mahato PK, Bhattacharyya S. Metabotropic glutamate receptor 1 recycles to the cell surface in protein phosphatase $2 \mathrm{~A}$-dependent manner in nonneuronal and neuronal cell lines. J Neurochem 2014; 131: 602-614.

52 Okabe S. Molecular anatomy of the postsynaptic density. Mol Cell Neurosci 2007; 34: $503-518$

53 Nair D, Hosy E, Petersen JD, Constals A, Giannone G, Choquet D et al. Superresolution imaging reveals that AMPA receptors inside synapses are dynamically organized in nanodomains regulated by PSD95. J Neurosci 2013; 33: 13204-13224.
54 Pougnet JT, Toulme E, Martinez A, Choquet D, Hosy E, Boué-Grabot E. ATP P2X receptors downregulate AMPA receptor trafficking and postsynaptic efficacy in hippocampal neurons. Neuron 2014; 83: 417-430.

55 El'sgol'ts LE, Norkin SB. Introduction to the theory of differential equations with deviating argument. Nauka: Moskow, 1971.

56 Likhoshvai VA, Kogai VV, Fadeev SI, Khlebodarova TM. On the relationship between the properties of one-dimensional maps of the control functions with the chaos in the equations of a special type with delayed argument. Math Biol Bioinform 2017; 12: 385-397.

57 Li TY, Yorke JA. Period three implies chaos. Amer Math Monthly 1975; 82: 985-992. 58 Rossler OE. An equation for hyperchaos. Phys Lett A 1979; 71: 155-157.

59 Benettin G, Galgani L, Giorgilli A, Strelcyn JM. Lyapunov exponents for smooth dynamical systems and Hamiltonian systems; a method for computing all of them, Part I: theory. Part 2: numerical applications. Meccanica 1980; 15: 9-30.

60 Benettin G, Galgani L, Strelcyn JM. Kolmogorov entropy and numerical experiments. Phys Rev A 1976; 14: 2338-2345.

61 Likhoshvai VA, Kogai VV, Fadeev SI, Khlebodarova TM. Chaos and hyperchaos in a model of ribosome autocatalytic synthesis. Sci Rep 2016; 6: 38870.

62 Khlebodarova TM, Kogai WV, Fadeev Sl, Likhoshvai VA. Chaos and hyperchaos in simple gene network with negative feedback and time delays. J Bioinf Comput Biol 2017: 15: 1650042 .

63 Likhoshvai VA, Fadeev Sl, Kogai V, Khlebodarova TM. On the chaos in gene networks. J Bioinform Comput Biol 2013; 11: 1340009.

64 Likhoshvai VA, Kogai VV, Fadeev Sl, Khlebodarova TM. Alternative splicing can lead to chaos. J Bioinform Comput Biol 2015; 13: 1540003.

65 Feigenbaum MJ. Universal behavior in nonlinear systems. Los Alamos Science 1980; 1: 4-27.

66 Feigenbaum MJ. The universal metric properties of nonlinear transformations. J Stat Phys 1979; 21: 669-706.

67 Canavier CC, Clark JW, Byrne JH. Routes to chaos in a model of a bursting neuron. Biophys J 1990; 57: 1245-1251

68 Mosekilde E, Lading B, Yanchuk S, Maistrenko Y. Bifurcation structure of a model of bursting pancreatic cells. Biosystems 2001; 63: 3-13.

69 Shen P, Larter R. Chaos in intracellular Ca2+ oscillations in a new model for nonexcitable cells. Cell Calcium 1995; 17: 225-232.

70 Martinez de la Fuente I, Martinez L, Veguillas J. Dynamic behavior in glycolytic oscillations with phase shifts. Biosystems 1995; 35: 1-13.

71 Kogai VV, Likhoshvai VA, Fadeev SI, Khlebodarova TM. Multiple scenarios of transition to chaos in the alternative splicing model. Int J Bifurcat Chaos 2017; 27: 1730006

(c) (\$) $\Theta$ This work is licensed under a Creative Commons Attributioncc) NonCommercial-NoDerivs 4.0 International License. The images or other third party material in this article are included in the article's Creative Commons license, unless indicated otherwise in the credit line; if the material is not included under the Creative Commons license, users will need to obtain permission from the license holder to reproduce the material. To view a copy of this license, visit http:// creativecommons.org/licenses/by-nc-nd/4.0/

(c) The Author(s) 2018

Supplementary Information accompanies the paper on the Molecular Psychiatry website (http://www.nature.com/mp) 